\title{
Avaliação do Desempenho de Gestores de Investimentos Sem Recurso a Carteiras Padrão
}

\author{
Carlos Machado-Santos \\ Manuel José da Rocha Armada
}

\section{RESUMO}

Em alternativa ao que tradicionalmente tem vindo a ser feito na área da avaliação do desempenho de gestores de carteiras de investimentos, alguns autores propuseram novas abordagens no sentido de se obterem medidas de performance, recorrendo à informação contida na composição dessas carteiras. Muito recentemente foram sugeridas novas metodologias que, para além de utilizarem como inputs a composição dos fundos a avaliar, não utilizam nenhum índice de mercado como carteira padrão, evitando desta forma a questão, largamente debatida e criticada na literatura, dos possíveis enviezamentos causados pela utilização de benchmarks ineficientes. Neste contexto, e com base numa amostra de seis fundos de investimento mobiliário para o mercado português, procedemos a um estudo na base da metodologia proposta por Grinblatt e Titman (1993). Os resultados indicam que os fundos obtiveram retornos globais positivos. Para os subperíodos estudados, a evidência empírica indica que os mesmos gestores parecem alcançar uma melhor performance quando o mercado accionista sofre variações negativas, o mesmo não se verificando quando este mercado varia positivamente e de forma acentuada.

Palavras-chaves: avaliação do desempenho de carteiras de investimentos, gestão de carteiras de investimentos.

\begin{abstract}
As an alternative to what traditionally has been made in the area of portfolio performance evaluation, some authors have proposed new approaches in order to obtain measures of performance using information included in the composition of such portfolios. Very recently, new methodologies have been proposed which, besides the use of the portfolios holdings as inputs, do not use any market index as a proxy, avoiding in this way the possible biases caused by the use of inefficient benchmarks that has been largely analyzed and discussed in the literature. In such a context, and with a database consisting of six professionally managed portfolios (mutual funds) of the portuguese market, we carried out a study based upon the methodology of Grinblatt and Titman (1993). The results show that those portfolios had positive overall returns. For the subperiods studied, the empirical evidence seems to indicate that the managers achieve a better performance when the stock market experiments negative returns, than when this market shows large positive returns.
\end{abstract}

Key words: portfolio performance evaluation, portfolio management. 


\section{INTRODUÇÃO}

A avaliação da performance dos gestores de investimentos sofreu uma enorme evolução nas últimas décadas, pelo que tem vindo a ser um dos tópicos mais importantes e actuais na área das finanças empresariais. A aceitação da Moderna Teoria da Carteira trouxe alterações profundas ao nível do processo de avaliação, que de simples cálculos de retornos passou a incorporar na avaliação não apenas o retorno mas também o nível de risco associado. Neste sentido, alguns autores (e.g.: Treynor, 1965; Sharpe, 1966; Jensen, 1968) desenvolveram medidas (tradicionais) de avaliação da performance ajustadas ao risco. Vários trabalhos empíricos baseados nestas medidas têm evidenciado persistentes níveis de performance inferior, pelo que a eficácia das mesmas tem sido largamente posta em causa (e.g.: Roll, 1978). Alternativamente a estas metodologias de avaliação da performance global, novas linhas de investigação foram entretanto surgindo (e.g.: Treynor e Mazuy, 1966; Pfleiderer e Bhattacharya, 1983; Lee e Rahman, 1990) no sentido de se avaliar a performance nos seus aspectos de timing e selectividade. Contudo, na sua maioria, estes estudos baseiam-se apenas em séries temporais de retornos.

Muito recentemente alguns autores (e.g.: Elton e Gruber, 1991) propuseram novas abordagens no sentido de isolar as componentes de timing e selectividade, recorrendo a informação específica dos títulos individuais que compõem as carteiras de investimentos, i.e., a partir da composição dessas carteiras. Por outras palavras, estas abordagens, ao contrário das anteriormente referidas consideram que, para além dos retornos observáveis dos fundos, existe informação adicional (composição dos fundos) disponível ao avaliador/observador externo.

Com base neste pressuposto e dadas as críticas às metodologias que requerem que o avaliador compare os retornos dos fundos com uma carteira padrão (e.g.: índice de mercado), Grinblatt e Titman (1993) desenvolvem uma nova medida que requer apenas a observação dos retornos dos títulos individuais em períodos consecutivos, os quais servem de padrão (em termos de comparação) para os períodos anteriores. Desta forma, os autores eliminam a necessidade de utilização de índices de mercado, tão largamente criticada na literatura.

Assim, o presente estudo pretende analisar a performance dos gestores de carteiras de investimentos, utilizando para o efeito a metodologia proposta por Grinblatt e Titman (1993), com base numa amostra de Fundos de Investimento Mobiliário em Portugal, relativamente à qual não se conhece outro estudo empírico, para além do realizado pelos próprios autores no trabalho acima mencionado. 


\section{Metodologias Baseadas em Séries Temporais de Retornos}

A primeira relação de equilíbrio que proporcionou o ajustamento dos retornos ao risco surgiu na década de 60 através do Modelo de Equilíbrio dos Activos Financeiros (CAPM) desenvolvido, fundamentalmente, por Sharpe (1964) e Lintner (1965). Por ser relativamente simples e intuitivo, o CAPM tornou-se um modelo de grande aceitação e vastamente utilizado, servindo de base às designadas medidas "tradicionais" de avaliação de performance. Treynor (1965) foi quem primeiro analisou a performance global, incorporando em simultâneo, e numa única medida, a rendibilidade e o risco, através do ratio que considera o retorno em excesso por unidade de risco sistemático. Sharpe (1966) propôs uma medida semelhante à de Treynor (1965), embora utilize o retorno em excesso por unidade de risco total. Contudo ambos os ratios propostos são medidas de performance relativa. Alternativamente, Jensen (1968) desenvolveu uma medida de performance absoluta, traduzida pelo $\boldsymbol{\alpha}$ da regressão baseada na versão ex-post do CAPM.

Apesar de amplamente utilizadas, as medidas tradicionais têm sido alvo de inúmeras críticas e objecções. Roll $(1978,1980,1981)$ foi um dos maiores críticos aos testes empíricos com a utilização do CAPM na avaliação da performance, argumentando que o recurso a proxies ${ }^{(1)}$ ineficientes leva ao enviezamento de quaisquer estimativas (consistentes e fiáveis) da Linha de Mercado de Títulos (SML), não sendo esta, portanto, apropriada para avaliar a performance das carteiras, conduzindo a critérios ambíguos. Lee e Jen (1978), Ferguson $(1980,1986)$ e Brown e Brown (1987) reforçam as críticas apontadas por Roll (1978, 1980, 1981) concluindo, no essencial, que a utilização de índices como proxies afectará os resultados empíricos do CAPM.

As medidas de avaliação de performance anteriormente analisadas, e mesmo alguns contextos teóricos de per si, revelam-se insuficientes dado que, no pressuposto que o nível de risco não se altera, avaliam apenas a capacidade do gestor em prever os preços dos títulos individuais (selectividade), ignorando as capacidades de timing eventualmente por este demonstradas. Na tentativa de distinguir estas duas capacidades, Treynor e Mazuy (1966) publicaram um trabalho pioneiro (de natureza fundamentalmente empírica) recorrendo, para o efeito, a uma regressão quadrática, em vez da relação linear proposta pelo CAPM. Foi, no entanto, Fama (1972) o primeiro autor a propor formalmente uma metodologia para a decomposição da performance nos seus aspectos de timing e selectividade, embora esta se revele de difícil aplicação empírica. Num outro trabalho, Jensen (1972) parte da correlação entre o retorno esperado e o retorno observado do mercado para obter medidas de timing, concluindo que não é possível obter, empiricamente, medidas separadas de timing e selectividade. Este trabalho é contestado por Grant (1977, 
1978), Admati e Ross (1985) e Dybvig e Ross (1985), que demonstram que a medida de Jensen (1972) - $\boldsymbol{\alpha}$ - pode resultar em performance inferior se as actividades de timing do gestor forem ignoradas.

Por seu lado, Kon e Jen $(1978,1979)$ examinam, através do método de switching regression, a possibilidade de existirem vários níveis de risco sistemático, criticando assim as estimativas obtidas pela regressão OLS que assume um beta estacionário. Também Fabozzi e Francis (1979) aplicam um procedimento alternativo, através de variáveis dummy, para analisar a variabilidade dos betas em épocas de alta e de baixa do mercado. Uma técnica similar foi testada por Alexander e Stover (1980), tendo estes obtido resultados semelhantes ao de Fabbozi e Francis (1979), pelo menos ao nível da não estacionariedade dos betas. Posteriormente, Chen e Stockum (1986) apontam alguns problemas à utilização de testes com variáveis dummy, essencialmente porque diferentes conceitos de mercado em alta e baixa podem conduzir a diferentes resultados, assim como criticam o trabalho de Kon e Jen $(1978,1979)$ porque estes atribuem actividades de timing à não estacionariedade dos betas. Para Chen e Stockum (1986), esta relação não é necessariamente verdadeira já que uma situação não implica obrigatoriamente a outra.

Em alternativa, Pfleiderer e Bhattacharya (1983) sugerem novas técnicas capazes de identificar separadamente, pelo menos em teoria, actividades de timing e selectividade. Corrigindo Jensen (1972) e baseados no estudo de Treynor e Mazuy (1966), desenvolvem uma técnica de regressão que requer apenas os valores observados dos retornos dos fundos e do mercado. Esta abordagem foi testada empiricamente nos Estados Unidos por Lee e Rahman (1990), por Armada (1992) no Reino Unido e por Cortez e Armada (1995) em Portugal.

Em síntese, de referir que as medidas de avaliação da performance até aqui analisadas $^{(2)}$ e baseadas apenas em séries temporais de retornos, para além das limitações anteriormente enunciadas, têm ainda evidenciado, ao nível dos estudos empíricos, uma persistente performance inferior dos gestores dos fundos de investimento e não mostram, salvo raras excepções, capacidades de timing do mercado.

\section{Metodologias Baseadas na Composição das Carteiras}

As medidas que utilizam, como inputs básicos, apenas séries temporais de retornos assumem claramente o pressuposto de simetria de informação por parte de quem gere as carteiras e de quem avalia a performance desses mesmos gestores. 
Contudo este pressuposto, como regra geral, não corresponde à realidade. As séries temporais de retornos não são, de per si, a única informação disponível ao avaliador para que este possa inferir sobre a performance, quer ao nível global quer ao nível do timing e selectividade. De facto, e para o efeito, é possível dispor de informação adicional, i.e., informação relativa à composição das carteiras.

Elton e Gruber (1991) desenvolvem um conjunto de medidas de performance capazes de identificar correctamente a informação contida nos fundos e que podem ser estimadas em termos de variáveis observáveis. Contrariamente às medidas baseadas em séries temporais de retornos, esta metodologia utiliza informação adicional disponível sobre as carteiras a serem avaliadas e permite medir isoladamente (pelo menos em teoria) o retorno global e as suas componentes de timing e de selectividade. Contudo a aplicação desta abordagem implica a utilização de um índice de mercado, pelo que grande parte das críticas debatidas na literatura e anteriormente apresentadas, se lhe podem também aplicar.

Cornell (1979), baseado na Event Study Measure, foi dos primeiros autores a defender a idéia da avaliação da performance sem recurso a carteiras padrão, propondo uma medida baseada na composição dos fundos, a qual avalia a performance através da diferença entre os retornos dos títulos quando incluídos numa carteira (no período t), com os retornos obtidos por esses mesmos títulos (fora do contexto da carteira) no período seguinte (período $t+1$ ). Contudo a escolha do período $t+1$ como proxy pode levar a enviezamentos devidos a problemas de "sobrevivência" dos títulos.

Muito recentemente, Grinblatt e Titman (1993) ultrapassam esta dificuldade com o desenvolvimento de uma nova metodologia - Portfolio Change Measure (PCM) - que, contrariamente a Cornell (1979), utiliza o período t-1 como proxy e substitui a diferença dos retornos dos títulos pela diferença dos "pesos" da carteira nos dois períodos consecutivos. Esta é uma das vantagens da PCM, já que, ao assumir uma perspectiva ex-post, permite trabalhar com dados conhecidos, utilizando apenas os retornos dos títulos que compõem a carteira no período de avaliação desta (período t). Por outro lado e como defendem os autores, em circunstância alguma podem os resultados ser atribuídos à utilização de um benchmark (carteira padrão) ineficiente.

\section{Metodologia e Descrição da Base de Dados}

\section{Metodologia}

Grinblatt e Titman (1989) mostraram que é possível ultrapassar os erros/pro- 
blemas que emergem da popular medida de Jensen (1968) através das designadas period weighting measures. Estas podem ser definidas, para uma amostra de $\mathrm{T}$ períodos, pela seguinte relação:

$$
\alpha=\sum_{t=1}^{T} W_{i, t} R_{i, t}
$$

onde $\mathrm{W}_{\mathrm{i}, \mathrm{t}}$ e $\mathrm{R}_{\mathrm{i}, \mathrm{t}}$ são, respectivamente, os pesos e os retornos da carteira a ser avaliada. Desta forma temos que, sendo $\mathrm{W}_{\mathrm{t}}>0$ para qualquer $t$, a medida de performance a converge, em probabilidade, para (a) zero para um investidor não informado e para (b) um valor positivo para um investidor com capacidade (informação) de selectividade mas não capacidade (informação) de timing. Definindo os pesos como:

$$
\mathrm{W}_{\mathrm{i}, \mathrm{t}}=\left(\sigma_{\mathrm{i}}^{2}-\left(\mathrm{R}_{\mathrm{i}, \mathrm{t}}-\overline{\mathrm{R}}_{\mathrm{i}}\right) \overline{\mathrm{R}}_{\mathrm{i}}\right) /\left(\mathrm{T} \sigma_{\mathrm{i}}^{2}\right)
$$

onde $\overline{\mathrm{R}}_{\mathrm{i}}$ e $\sigma_{\mathrm{i}}^{2}$ são a média e variância, para a amostra, dos retornos da carteira, os autores mostram que, desta forma, a medida de Jensen (1968) pode ser expressa através da seguinte relação:

$$
\alpha_{i}=\sum_{t=1}^{T} W_{i, t} R_{i, t}=\bar{R}_{i}-\beta_{i} \bar{R}_{m}
$$

onde $a_{i}$ é a recta resultante da regressão de $R_{i, t}$ sobre $R_{m, t}$. Grinblatt e Titman (1989) restringiram os pesos a $\mathrm{W}_{t}>0$ por forma a evitar que a medida de Jensen (1968) fosse negativa quando o investidor/gestor possui informação de timing. Contudo e como demonstram Cumby e Glen (1990), os resultados desta medida não são fiáveis quando as informações de selectividade e timing são correlacionadas, invalidando desta forma a restrições impostas para os pesos. 
Posteriormente, Grinblatt e Titman (1993), reconhecendo os argumentos que limitam a aplicabilidade destas medidas, mostram que conhecendo a composição da carteira a ser avaliada, o avaliador pode eliminar a necessidade de comparar os retornos com uma carteira padrão (e.g.: índice de mercado), evitando assim todos os problemas que lhe são inerentes. Apoiados no estudo de Cornell (1979) os autores derivam uma medida que é uma adaptação da Event Study Measure (EMS) cuja relação tem a seguinte forma:

$$
\mathrm{ESM}=\frac{1}{\mathrm{~T}} \sum_{\mathrm{t}=1}^{\mathrm{T}} \sum_{\mathrm{i}=1}^{\mathrm{N}}\left[\mathrm{W}_{\mathrm{i}, \mathrm{t}}\left(\mathrm{R}_{\mathrm{i}, \mathrm{t}}-\mathrm{R}_{\mathrm{i}, \mathrm{t}+1}\right)\right]
$$

Como se pode verificar, esta abordagem avalia a performance através da diferença entre os retornos dos títulos quando incluídos numa carteira (o período do "evento" $t$ ), com os retornos obtidos por esses mesmos títulos numa data posterior (período de comparação $t+1$ ). Contudo a escolha do período $t+1$ como proxy pode levar a enviezamentos devidos a problemas de sobrevivência dos títulos, o que leva os autores a derivar uma nova metodologia que, embora baseada nos mesmos princípios, utiliza o período t-1 como proxy e substitui a diferença dos retornos dos títulos pela diferença dos "pesos" da carteira nos dois períodos consecutivos. A medida proposta por estes autores - Portfolio Change Measure (PCM) - pode ser expressa pela seguinte relação ${ }^{(3)}$ :

$$
\mathrm{PCM}=\frac{1}{\mathrm{~T}-1} \sum_{\mathrm{t}=1}^{\mathrm{T}} \sum_{\mathrm{i}=1}^{\mathrm{N}}\left[\mathrm{R}_{\mathrm{i}, \mathrm{t}}\left(\mathrm{W}_{\mathrm{i}, \mathrm{t}}-\mathrm{W}_{\mathrm{i}, \mathrm{t}-1}\right)\right]
$$

onde, $\mathrm{R}_{\mathrm{i}, \mathrm{t}}$ é o retorno em excesso do activo de risco i no período $\mathrm{t}, \mathrm{W}_{\mathrm{i}, \mathrm{t}}$ é o peso do activo de risco i no período $\mathbf{t} \mathrm{e}$ as restantes variáveis conforme definição anterior. Os pesos são dados pela equação [2] anteriormente expressa. Neste caso, não se calcula o peso da carteira no seu todo, mas sim os pesos de todos os títulos individuais incluídos nesta. Como tal, a soma dos pesos para cada título e ao longo dos $\mathrm{T}$ períodos deve igualar a unidade:

$$
\sum_{\mathrm{t}=1}^{\mathrm{T}} \mathrm{W}_{\mathrm{i}, \mathrm{t}}=1 \quad \quad \text { e, obviamente, } \quad \sum_{\mathrm{t}=1}^{\mathrm{T}} \sum_{\mathrm{i}=1}^{\mathrm{N}} \mathrm{W}_{\mathrm{i}, \mathrm{t}}=\mathrm{N}
$$


Ou seja, a soma dos pesos individuais dos títulos que compõem a carteira no período em análise deve igualar o número total desses mesmos activos. De notar que, contrariamente às abordagens anteriores, a PCM permite trabalhar com dados conhecidos (ao assumir uma perspectiva ex-post), o que se traduz numa das principais vantagens desta metodologia. Pode também verificar-se que, basicamente, a informação requerida para a análise se traduz pelo conhecimento do número de acções que compõem a carteira, das proporções investidas e dos retornos de cada um destes activos de risco. De salientar que os resultados obtidos através da PCM, dados os pressupostos apresentados, não podem ser, em quaisquer circunstâncias, atribuídos a índices ineficientes, dada a não utilização destes. Desta forma, e como já foi referido, Grinblatt e Titman (1993) pretendem também ultrapassar a questão dos benchmarks ineficientes largamente debatida na literatura.

\section{Descrição da Base de Dados}

A amostra é constituída por seis Fundos de Investimento Mobiliário para o período de Janeiro de 1990 a Dezembro de 1994, do qual resulta um total de 120 observações para cada fundo e cada título individual. Estes fundos foram seleccionados, em primeiro lugar, tendo em conta o facto de serem considerados accionistas. Por outro lado, para um horizonte temporal alargado, são poucos os fundos com informação disponível para o período em referência (dado que este mercado é relativamente recente), justificando-se desta forma o número de fundos incluído na amostra. Por último, uma terceira razão prende-se com o facto destes fundos terem sido objecto de análise em dois estudos anteriormente realizados por Cortez e Armada (1995) e Santos e Armada (1996), relativamente a um horizonte temporal idêntico, podendo-se assim comparar os resultados obtidos com os do estudo agora elaborado.

A composição dos fundos foi obtida junto das respectivas Sociedades Gestoras e através dos Boletins Oficiais das Bolsas de Valores de Lisboa e Porto ${ }^{(4)}$. A informação observada para os fundos contém, para cada período, dados relativos a todos os títulos que os compõem, i.e., a cotação, quantidade e respectivo valor de mercado de cada activo de risco e os valores totais investidos nos designados activos sem risco. Os retornos ajustados a dividendos, para cada título e período quinzenal, foram calculados de acordo com a seguinte expressão:

$$
\mathrm{R}_{\mathrm{i}, \mathrm{t}}^{\prime}=\left(\left(\mathrm{P}_{\mathrm{i}, \mathrm{t}}+\mathrm{D}_{\mathrm{i}, \mathrm{t}}\right)-\mathrm{P}_{\mathrm{i}, \mathrm{t}-1}\right) / \mathrm{P}_{\mathrm{i}, t-1}
$$


onde $\mathrm{R}_{\mathrm{i}, \mathrm{t}}$ é o retorno do título i no período $\mathrm{t}, \mathrm{P}_{\mathrm{i}, \mathrm{t}}$ é o preço (cotação) de mercado do título i no período te $\mathrm{D}_{\mathrm{i}, \mathrm{t}}$ os dividendos distribuídos pelo título i no período t. Para a taxa isenta de risco foram consideradas as taxas médias ponderadas dos Bilhetes do Tesouro (BT's) a 91 dias, obtidos junto do Banco de Portugal. A taxa quinzenal foi calculada através da relação de proporcionalidade. Desta forma, o retorno em excesso $\mathrm{R}_{\mathrm{i}, \mathrm{t}}$ foi calculado segundo a expressão:

$$
\mathrm{R}_{\mathrm{i}, \mathrm{t}}=\mathrm{R}_{\mathrm{i}, \mathrm{t}}^{\prime}-\mathrm{R}_{\mathrm{f}, \mathrm{t}}
$$

onde $\mathrm{R}_{\mathrm{i}, \mathrm{t}}$ é o retorno em excesso do título i no período $\mathrm{t}$ e $\mathrm{R}_{\mathrm{f}, \mathrm{t}}$ a taxa isenta de risco (BT's) no período $\mathbf{t}$.

A Tabela 1 a seguir apresenta uma síntese das principais características dos FIM da amostra.

Tabela 1: Resumo das Características da Base de Dados Jan. 1990 a Dez. 1994

\begin{tabular}{|lccc|}
\hline Nome Fundos & Períodos Quinzenais & Total Acções & Total Observações \\
\hline FIP & 120 & 88 & 10560 \\
INV & 120 & 111 & 13320 \\
MUL & 120 & 73 & 8760 \\
PRI & 120 & 83 & 9960 \\
UNI & 120 & 92 & 11040 \\
VAL & 120 & 42 & 5040 \\
\hline
\end{tabular}

O número total das acções para os seis fundos é de 147, 19 das quais não nacionais. Como tal, parte destas acções fazem parte, em simultâneo, dos diferentes fundos. De referir que à excepção do fundo VAL, que detém o menor número destes títulos, chegando a ter apenas o total de 6 no período de 1992 e de 8 no período de 1993 (Anexo 2), os restantes fundos são relativamente mais diversificados. Contudo parece que estes gestores se especializaram num número limitado de indústrias, como se pode verificar pela análise da proporção investida, por exemplo, nos bancos (e.g.: BCM e BCP) e nas comunicações (e.g.: Marconi). De facto, verificou-se que os títulos destes sectores estão presentes em todos os fundos e assumem grande parte do investimento nos activos de risco. 
De notar também que não existe uma ligação muito directa entre o número de acções por fundo e o valor (montante) correspondente. Para o período de 1990 a 1994, os fundos INV, FIP e VAL detêm, respectivamente e em simultâneo, o primeiro, segundo e sexto lugar quer no número de acções quer no montante em valor de mercado (Anexos 2 e 3). Por outro lado, o fundo UNI, em segundo no ranking do número de acções, aparece apenas em quarto lugar relativamente ao valor investido nestes títulos. Situação relativamente idêntica verifica-se com os fundos PRI e MUL.

Para os restantes períodos destaca-se o ano de 1990, no qual o fundo INV, com um valor médio aproximado dos 3.355 biliões de escudos, ultrapassa em cerca de 10 vezes o valor investido pelo fundo VAL e duas vezes o fundo FIP (sexto e segundo, respectivamente, no ranking dos montantes investidos). Nos anos de 1991, 1992 e 1993 essa diferença, embora elevada, vai-se diluindo e em 1994 os valores invertem-se, sendo o fundo INV, por um lado ultrapassado pelo fundo VAL e, por outro, verifica-se que neste período é o fundo que menos investiu em acções. Desta forma e em síntese, verifica-se (1) uma grande variabilidade nos montantes investidos em acções, por fundo, ao longo do período global em análise e (2) que na sua maioria os fundos desinvestiram ao longo dos primeiros 3 anos da amostra, tendo-se recuperado nos dois anos seguintes (Anexos 2 e 3). Excepções são o fundo INV que desinvestiu sistematicamente e de forma acentuada durante os cinco anos, passando de uma média de 3.356 biliões de escudos em 1990 para um valor de apenas 348 milhões de escudos em 1994 (uma redução de 89.6\%), e o fundo FIP que manteve ao longo dos cinco anos um investimento de montante relativamente estável.

\section{EVIDÊnCIA Empírica}

\section{Análise para o período global (1990-1994)}

As estimativas de performance global obtidas através da medida expressa na equação [5] - PCM - para os seis fundos de investimento, são apresentadas na Tabela 2 a seguir.

Verifica-se que os fundos apresentam estimativas de performance positivas, variando entre um máximo de $0.033 \%$ e um mínimo de $0.0106 \%$ (valores quinzenais). Para inferir a significância estatística da medida de performance, procedeuse a uma análise dos valores "t-stat" (5) apresentados na tabela a seguir. Pela sua observação, constata-se que estes valores são superiores a $1.658^{(6)}$, sendo por isso significativamente positivos a um nível de $5 \%$, o que leva a rejeitar a hipótese nula da performance (PCM) ser igual a zero. Neste contexto, tais resultados indi- 
cam que, em média e ao longo dos 5 anos, os fundos geraram retornos positivos, apesar da instabilidade do mercado e das elevadas taxas dos BT's. De facto, conforme se pode ver nos Anexos 4 e 5, o retorno médio (quinzenal) do índice BVL foi, para o período de 1990 a 1994, de 0.03094\% (desvio padrão de $2.87147 \%$ ). Para o mesmo período e mesma frequência, a taxa dos BT's foi de $0.61248 \%$ (desvio padrão de $0.12414 \%$ ).

Tabela 2: Estimativas de Performance dos Fundos - 1990-1994

\begin{tabular}{|lccc|}
\hline Fundo & Ranking & Performance & t-stat \\
\hline FIP & 4 & $0.0238 \%$ & 3.071 \\
INV & 1 & $0.0330 \%$ & 2.044 \\
MUL & 3 & $0.0267 \%$ & 2.815 \\
PRI & 2 & $0.0299 \%$ & 2.909 \\
UNI & 5 & $0.0154 \%$ & 1.850 \\
VAL & 6 & $0.0106 \%$ & 1.977 \\
\hline
\end{tabular}

De uma análise das estimativas de performance, destaca-se o fundo INV que ao alcançar, em média e ao longo dos cinco anos, a melhor performance da amostra, o parece ter conseguido através de uma escolha correcta e diversificada de títulos, que se reflecte na composição da sua carteira. De facto este fundo apresenta o montante mais elevado de acções, em número e valor de mercado (Anexos 2 e 3). Além disso, à excepção do investimento médio feito nas acções da Soporcel (indústria de pasta de papel) que representa $20.34 \%$ do total investido por este fundo em acções, o restante do investimento por cada acção individualmente é relativamente homogéneo. Destaque-se ainda o facto deste ter sido o único fundo a realizar aplicações em acções não nacionais, com um valor de cerca $6.87 \%$ do total investido nos activos de risco.

Por contraste, destaca-se o fundo VAL que ao alcançar, em média e ao longo dos cinco anos, a performance mais fraca da amostra, parece ter sido punido por uma escolha pouco diversificada de títulos. De facto, este fundo apresenta um montante reduzido de acções, em número e valor de mercado (Anexos 2 e 3), para além de que concentrou a quase totalidade das suas aplicações em activos de risco essencialmente em quatro sectores: Banca (43.59\%), Distribuição (26.95\%), Comunicações $(10.74 \%)$ e Leasing $(5.89 \%)$. Por outro lado, dentro de cada sector existe outra concentração, sendo os totais acima referidos respeitantes apenas a um pequeno número de empresas. Assim, verifica-se que este fundo investiu, em 
média e ao longo do período da amostra, basicamente em 6 empresas, com proporções que vão desde $19.19 \%$ (BCP) a $5.88 \%$ (Lusoleasing). Curiosamente, todas estas acções apresentaram retornos médios negativos nos anos de 1990, 1991 e 1992, o que confirma os resultados menos favoráveis obtidos. Para os restantes fundos as estimativas de performance conseguidas parecem estar mais de acordo com a evolução dos montantes investidos, em número e valor de mercado, nos activos de risco. Excepção talvez para o fundo UNI, que parece ter sido castigado, em grande medida, pela extremamente fraca performance conseguida no período de 1993, conforme veremos adiante.

\section{Análise para os subperíodos de 1990-1992 e 1993-1994}

A escolha destes subperíodos de análise teve a ver, fundamentalmente, com a evolução do índice BVL ao longo dos cinco anos do período global. Recorde-se que o índice BVL apresentou, em média, retornos quinzenais negativos para os anos de 1990, 1991 e 1992, conseguindo retornos positivos em 1993 e 1994 (Anexo 5). Pretende-se assim comparar a performance dos gestores numa época de evolução genericamente desfavorável do mercado accionista, com um período (posterior) em que este mercado foi mais favorável. Por outro lado, conforme referido anteriormente, esta escolha permitirá melhor comparação com os resultados de Santos e Armada (1996), os quais analisaram também estes subperíodos. As estimativas de performance global para estes dois subperíodos são apresentadas na Tabela 3 a seguir.

Para o primeiro subperíodo, os resultados mostram que todos os fundos apresentam estimativas de performance global positivas, bastante superiores às do período global, variando entre um máximo de $0.0972 \%$ e um mínimo de $0.0206 \%$. Note-se, uma vez mais, que estes valores são estatisticamente significativos a um nível de 5\%, conforme se pode verificar pelos valores "t-stat" apresentados. A ordenação dos fundos neste subperíodo é bastante semelhante à do período global, com excepção para o fundo UNI, cuja performance aumentou de $0.0154 \%$ para $0.0952 \%$, passando de quinto para segundo no ranking dos fundos. De facto, comparando a ordenação dos fundos, segundo a sua performance, nestes dois períodos, verifica-se a existência de uma correlação ${ }^{(7)}$ positiva de cerca de $60 \%$ (Anexos 6 e 7).

Para o segundo subperíodo verifica-se que, à excepção de dois fundos, os restantes apresentam estimativas de performance negativas (embora nenhuma destas estimativas seja estatisticamente significativa a um nível de 5\%). Contrariamente, os fundos INV e VAL apresentam estimativas de performance positivas, significativas para o mesmo nível de confiança. É curioso observar que a ordenação dos 
fundos neste subperíodo é substancialmente diferente da dos períodos anteriores, onde se registam valores de $r_{s}$, respectivamente, de $14.29 \%$ e $-25.71 \%$ (Anexos 6 e 7). As alterações ocorridas devem-se, em grande parte, à diferente ordenação dos fundos UNI e VAL, sendo para os restantes relativamente estável. De notar que o fundo VAL consegue as melhores estimativas de performance, o que se poderá explicar também pelo reforço do montante investido em acções numa fase mais favorável do mercado accionista. De facto, foi este o fundo que mais de perto seguiu o crescimento do mercado, tendo sido mesmo o único a aumentar o investimento em acções em 1993, durante o qual o índice BVL exibiu, como já referimos, rendibilidade elevada (Anexos 3 e 5).

Tabela 3: Estimativas de Performance dos Fundos - 1990-1992 e 1993-1994

\begin{tabular}{|c|c|c|c|c|c|c|}
\hline \multirow[b]{2}{*}{ Fundo } & \multicolumn{3}{|c|}{ 1990-1992 } & \multicolumn{2}{|c|}{ 1993-1994 } & \\
\hline & Ranking & Performance & t-stat & Ranking & Performance & t-stat \\
\hline FIP & 4 & $0.0801 \%$ & 3.187 & 5 & $-0.0033 \%$ & -1.530 \\
\hline INV & 1 & $0.0972 \%$ & 1.915 & 2 & $0.0026 \%$ & 1.740 \\
\hline MUL & 5 & $0.0757 \%$ & 4.221 & 4 & $-0.0021 \%$ & -1.061 \\
\hline PRI & 3 & $0.0887 \%$ & 3.360 & 3 & $-0.0015 \%$ & -0.656 \\
\hline UNI & 2 & $0.0952 \%$ & 3.667 & 6 & $-0.0123 \%$ & -1.266 \\
\hline VAL & 6 & $0.0207 \%$ & 1.817 & 1 & $0.0699 \%$ & 2.021 \\
\hline
\end{tabular}

\section{Análise para subperíodos anuais}

Dada a alteração acentuada na ordenação das carteiras, conforme o intervalo de tempo (período) utilizado para o cálculo da performance ${ }^{(8)}$, e a disparidade das estimativas de performance alcançadas por parte de alguns dos fundos, pareceunos importante subdividir o período global em períodos anuais, o que permitirá uma análise adicional do comportamento das carteiras da amostra. Neste contexto, as estimativas de performance para os períodos de 1990, 1991, 1992, $1993 \mathrm{e}$ 1994, são apresentadas nas Tabelas 4 e 5 a seguir: 
Tabela 4: Estimativas de Performance dos Fundos - 1990, 1991 e 1992

\begin{tabular}{|c|c|c|c|c|c|c|c|c|c|}
\hline \multirow[b]{2}{*}{ Fundo } & . & 1990 & & \multicolumn{2}{|r|}{1991} & & \multicolumn{2}{|r|}{1992} & - \\
\hline & Ranking & Performance & t-stat & Ranking & Performance & t-stat & Ranking & Performance & t-stat \\
\hline FIP & 4 & $0.3305 \%$ & 2.150 & 4 & $0.1636 \%$ & 2.458 & 3 & $0.2294 \%$ & 1.867 \\
\hline INV & 1 & $0.4852 \%$ & 3.286 & 3 & $0.1759 \%$ & 1.718 & 2 & $0.2327 \%$ & 3.334 \\
\hline MUL & 5 & $0.2601 \%$ & 4.751 & 5 & $0.1571 \%$ & 2.560 & 1 & $0.2998 \%$ & 2.813 \\
\hline PRI & 2 & $0.4453 \%$ & 4.050 & 2 & $0.1954 \%$ & 1.886 & 4 & $0.2076 \%$ & 2.918 \\
\hline UNI & 3 & $0.4400 \%$ & 2.387 & 1 & $0.2798 \%$ & 3.200 & 5 & $0.1561 \%$ & 2.793 \\
\hline VAL & 6 & $0.1581 \%$ & 2.766 & 6 & $0.0394 \%$ & 1.796 & 6 & $0.0189 \%$ & 2.315 \\
\hline
\end{tabular}

Tabela 5: Estimativas de Performance dos Fundos - 1993 e 1994

\begin{tabular}{|c|c|c|c|c|c|c|}
\hline Fundo & Ranking & $-\frac{1993}{\text { Performance }}$ & -- & Ranking & $\frac{1994}{-}-$ & -- \\
\hline FIP & 5 & $-0.0213 \%$ & -1.994 & 4 & $0.0863 \%$ & 2.150 \\
\hline INV & 1 & $0.0302 \%$ & 1.977 & 6 & $0.0307 \%$ & 3.286 \\
\hline MUL & 4 & $-0.0203 \%$ & -1.184 & 2 & $0.1368 \%$ & 4.751 \\
\hline PRI & 3 & $-0.0175 \%$ & -1.026 & 1 & $0.1773 \%$ & 4.050 \\
\hline UNI & 6 & $-0.0392 \%$ & -1.552 & 5 & $0.0607 \%$ & 2.387 \\
\hline VAL & 2 & $0.0006 \%$ & 0.809 & 3 & $0.0921 \%$ & 2.766 \\
\hline
\end{tabular}

Numa primeira observação das tabelas acima, verifica-se que as estimativas de performance são genericamente superiores às conseguidas nos períodos analisados anteriormente. Por outro lado, é de salientar que, à excepção de 1993, os fundos conseguiram retornos significativamente positivos a um nível de $5 \%$. Notase também que, especialmente nos anos de 1990, 1991 e 1992, período em que o mercado accionista obteve rendibilidades médias negativas, a performance dos 
fundos é claramente superior aos dois anos seguintes (1993 e 1994), período em que o mercado accionista obteve rendibilidades médias positivas.

Como para os primeiros três anos da amostra se verifica uma performance positiva e estatisticamente significativa para todos os fundos, seja qual for o horizonte temporal escolhido para análise (veja-se que a correlação da ordenação dos fundos - Anexo 7 - é bastante elevada para estes períodos), será curioso observar o comportamento destes fundos nos dois últimos anos. Um facto que ressalta desde logo é que a performance do período global de 1990-1994 e subperíodo de 1993-1994 é negativamente afectada pelos fracos resultados alcançados no ano de 1993, cujas estimativas de performance, à excepção do fundos INV e VAL, são negativas (embora apenas dois desses valores tenham significância a um nível de $5 \%$ ). De relembrar que neste período o mercado accionista registou uma subida, em termos anualizados, de cerca de 44\% (Anexo 5).

No ano de 1994 parece que os fundos conseguiram recuperar-se, tendo conseguido registar de novo estimativas de performance positivas (estatisticamente significativas), embora com valores inferiores aos alcançados nos anos de 1990 a 1992, já que a performance varia entre um máximo de $0.17725 \%$ para o fundo PRI e um mínimo de $0.03075 \%$ para o fundo INV. Verifica-se também que neste período houve um reforço de investimento em acções, o que poderá ajudar a explicar a recuperação observada pelos fundos.

O fundo INV é de certa forma uma excepção. Manteve praticamente a mesma performance nestes dois anos, com uma diferença para menos de apenas $0.00058 \%$ de 1993 para 1994. No entanto no ranking passou de primeiro para último. Este fundo, contrariamente aos restantes, desinvestiu neste segundo período, passando de um montante em acções de aproximadamente 636 milhões de escudos em 1993, para apenas 348 milhões (cerca de metade) em 1994. Daqui resulta que a penalização e consequente alteração no ranking se deve essencialmente ao facto do INV não ter acompanhado os resultados alcançados pelos restantes fundos, para além de ajudar a explicar a correlação quase inexistente entre a ordenação dos fundos em 1993 e 1994 (Anexo 7).

Em síntese, da observação das estimativas obtidas ao longo dos cinco anos da amostra, quer analisados num período único quer em períodos parciais, a evidência empírica sugere que os gestores destes fundos alcançaram performances positivas, à excepção do ano de 1993 (no qual o mercado accionista esteve particularmente favorável) em que a performance de quatro dos seis fundos se mostrou, na generalidade, significativamente negativa. 


\section{Análise comparativa com estudos anteriores}

Como referimos, um dos pontos de interesse será o de comparar os resultados de performance obtidos através das metodologias tradicionais, que utilizam apenas séries temporais de retornos, com as estimativas de performance resultantes da aplicação das mais recentes metodologias que contemplam a composição dos fundos.

Cortez e Armada (1995) realizaram um estudo no qual aplicaram as medidas tradicionais de Treynor (1965), Sharpe (1966), Jensen (1968) e as mais recentes de Pfleiderer e Bhattacharya (1983) - posteriormente melhoradas por Lee e Rahman (1990) - a uma amostra de sete ${ }^{(9)}$ fundos de investimento, em relação ao mercado Português, para os períodos de Março de 1989 a Fevereiro de 1993, tendo os resultados obtidos mostrado estimativas de performance negativas, tal como, de resto, a evidência empírica internacional também tem mostrado relativamente à aplicação destas abordagens. Uma das razões frequentemente apontada para este aparente insucesso dos fundos tem origem nas próprias medidas, uma vez que estas não utilizam informação adicional disponível ao avaliador (e.g.: a composição das carteiras).

Neste contexto, Hwang (1988) aplicou, pela primeira vez, a metodologia de Elton e Gruber (1991), a qual contempla a composição dos fundos, a uma amostra de 5 fundos em relação ao mercado dos Estados Unidos, para um período aproximado de quatro anos, tendo os resultados revelado estimativas de performance positivas. De seguida e mais recentemente, Santos e Armada (1996) aplicaram a referida metodologia de Elton e Gruber (1991) aos seis fundos de investimento da amostra utilizada no presente estudo e para o mesmo período de Janeiro de 1990 a Dezembro de 1994. Os resultados, idênticos aos agora conseguidos, mostram estimativas de performance com valores positivos (significativos para um nível de 5\%) para todos os fundos, para o período global de 19901994, assim como para o subperíodo de 1990-1992. Por outro lado, para o subperíodo de 1993-1994, quatro dos fundos apresentaram estimativas de performance negativas e os restantes dois fundos performances positivas. Tais valores parecem reforçar os resultados agora alcançados.

\section{CONCLUSÓES}

Os resultados obtidos sugerem que, para o período global de Janeiro de 1990 a Dezembro de 1994, todos os fundos da amostra conseguiram retornos em excesso positivos. 
Para o primeiro subperíodo, Janeiro de 1990 a Dezembro de 1992, durante o qual o mercado accionista foi genericamente menos favorável, os resultados mostram, uma vez mais, que estes fundos apresentam estimativas de performance positivas, e estatisticamente significativas. Por sua vez, a performance dos fundos aumentou significativamente neste subperíodo e para os seis fundos.

Para o segundo subperíodo, Janeiro de 1993 a Dezembro de 1994, durante o qual o mercado accionista foi genericamente mais favorável, verifica-se que à excepção de dois fundos, os restantes apresentam retornos globais negativos.

Quando analisados em períodos anuais, os fundos apresentam resultados idênticos ao período global, com estimativas de performance positivas, exceptuando o ano de 1993, em que a performance de quatro fundos é bastante negativa, o que ajuda a explicar a fraca prestação destes fundos quando analisados no período agregado de 1993-1994.

Neste contexto, e da observação das estimativas obtidas ao longo dos cinco anos em análise e para os vários períodos estudados, a evidência empírica parece sugerir que os gestores destes fundos conseguem melhores performances quando o mercado accionista sofre variações negativas, o mesmo não se verificando quando este mercado varia positivamente e de forma acentuada.

Por último, observamos que estes resultados são idênticos aos obtidos por Hwang (1988) e Santos e Armada (1996), que aplicaram metodologias de avaliação de performance que contemplam a composição das carteiras. Contudo os nossos resultados divergem dos de Cortez e Armada (1995), os quais obtiveram estimativas de performance negativas, ao aplicar medidas que utilizam apenas séries temporais de retornos medidos através das unidades de participação dos fundos.

Em síntese, torna-se importante realçar que a aplicação da metodologia de Grinblatt e Titman (1993), como já se tinha verificado, para a mesma amostra, com a metodologia de Elton e Gruber (1991), ao utilizar informação adicional disponível sobre a composição das carteiras, parece tender a produzir estimativas de performance mais encorajadoras para a gestão profissional dos fundos. Por outro lado, ao não utilizar o índice de mercado como carteira padrão, esta metodologia parece conseguir ultrapassar a questão dos erros causados por benchmarks ineficientes.

\section{NOTAS}

\footnotetext{
* A editoria preferiu manter a ortografia do original dos autores lusos.

${ }^{1}$ Em substituição da carteira de mercado que, segundo o CAPM, deveria ser o padrão natural de comparação.
} 
${ }^{2}$ Quer as clássicas, quer as mais recentes que identificam as capacidades de timing e selectividade do gestor.

${ }^{3}$ Aqui somos forçados a introduzir uma pequena alteração relativamente à equação proposta pelos autores. Grinblatt e Titman (1993) dividem o produto resultante dos somatórios por T. Contudo, em termos empiricos, torna-se necesario utilizar T-1 dado que $\mathrm{T}$ períodos implicam, obvia e obrigatoriamente, $\mathrm{T}-1$ intervalos (ver esquema operacional de cálculo no Anexo 1).

${ }^{4}$ A duplicidade de informação recolhida serviu para uma verificação minuciosa da composição da carteira (valor dos títulos individuais) para todos os fundos e períodos. Dado que a legislação permitia, até finais de 1994, um ajuste de 5\% para mais ou menos nos preços dos títulos, o valor das acções contidas nos fundos nem sempre reflectia a sua cotação real. Desta forma, e para garantir a fiabilidade dos resultados, sempre que estes valores não coincidiam, foi utilizado o preço de mercado.

${ }^{5}$ Estes valores foram calculados de acordo com Grinblatt e Titman (1993), dividindo o retorno médio de todos os títulos e períodos (quinzenais) pelo erro padrão da média.

${ }^{6}$ O valor "t-stat" para um nível de significância de 0.05 e com 119 graus de liberdade, é aproximadamente 1.658.

${ }^{7}$ A correlação foi calculada recorrendo à medida de correlação de ordenações - Spearman's Rank Correlation Coefficient $\left(\mathrm{r}_{\mathrm{s}}\right)$, cuja fórmula é dada pela seguinte expressão:

$$
\mathrm{r}_{\mathrm{s}}=1-\left(6 \sum_{\mathrm{i}=1}^{\mathrm{n}}\left(\mathrm{X}_{\mathrm{i}}-\mathrm{Y}_{\mathrm{i}}\right)^{2} / \mathrm{n}\left(\mathrm{n}^{2}-1\right)\right)
$$

onde $\mathrm{n}$ é o número de valores a ordenar, $\mathrm{X}_{\mathrm{i}}$ é a ordem da iésima variável $\mathrm{X}$, e $\mathrm{Y}_{\mathrm{i}}$ é a ordem da iésima variável $\mathrm{Y}$.

${ }^{8}$ Este problema colocado relativamente às medidas tradicionais de avaliação da performance parece não poder ser ainda ultrapassado. De facto, a escolha de diferentes períodos para análise tende uma vez mais a alterar significativamante a ordenação dos fundos, o que parece estar de acordo com as objecções largamente debatidas na literatura (e.g.: Fielitz e Greene, 1980; Levy, 1981, 1984).

${ }^{9}$ De referir que seis desses fundos fazem parte da presente amostra.

\section{ReferênCIAs Bibliográficas}

ADMATI, A.;

ROSS, S.

Measuring investment performance in a rational expectations equilibrium model. Journal of Business, v. 58 , n. 1 , p.01-26, 1985.

\section{ALEXANDER, G. J.;}

STOVER, R. D.

Consistency of mutual fund performance during varying market conditions. Journal of Economics and Business, p.219226, 1980.

ARMADA, M. J. R.

On the investigation of timing and selectivity in portfolio management. Manchester, 1992. Dissertation (Ph.D.) - Manchester Business School.
BROWN, K. C.;

BROWN, G. D.

Does the composition of the market portfolio really matter? Journal of Portfolio Management, p.26-32, 1987.

CHEN, C. R.;

STOCKUM, S.

Selectivity, market timing, and random beta behaviour of mutual funds : a generalised model. Journal of Financial Research, v.9, n.1, p.87-96, Spring 1986.

CORNELL, B.

Asymmetric information and portfolio performance measurement. Journal of Financial Economics, v.7, p.381-390, 1979 . 
CORTEZ, M. C. R.;

ARMADA, M. J. R.

Sobre a avaliação da performance de fundos de investimento. In: XIX ENCONTRO ANUAL DA ANPAD (1995 : Salvador). Anais ... ANPAD : Salvador, 1995.

CUMBY, R. E.;

GLEN, J. D.

Evaluating the performance of International Mutual Funds. Journal of Finance, v.45, n.2, p.497-521, June 1990.

DYBVIG, P. H.;

ROSS, S. A.

Differential information and performance measurement using a security market line. Journal of Finance, v.15, n.2,p.383-398, 1995.

ELTON, E. J.;

GRUBER, M. J.

Differential information and timing ability. Journal of Banking and Finance, v.15, p.117-131, 1991.

FABOZZI, F. J.;

FRANCIS, J. C.

Mutual fund systematic risk for bull and bear markets : an empirical examination. Journal of Finance, v.34, n.5, p.1243-1250, 1979.

FAMA, E. F.

Components of investment performance. Journal of Finance, v.27, n.3, p.551-567, June 1972.

FERGUSON, R.

Performance measurement doesn't make sense. Financial Analysts Journal, p.59-69, May/ June 1980.

The trouble with performance measurement. Journal of Portfolio Management, p.04-09, Spring 1986.
FIELITZ, B. D.;

GREENE, M. T.

Shortcomings in portfolio evaluation via MPT. Journal of Portfolio Management, p.13-19, 1980.

GRANT, D.

Market timing and portfolio management. Journal of Finance, v.33, n.4, p.1119-1131, Sept. 1978.

Portfolio performance and the "cost" of timing decisions. Journal of Finance, v.32, n.3, p.837-845, June 1977.

GRINBLATT, M.;

TITMAN, S.

Mutual fund performance : an analysis of quarterly portfolio holdings. Journal of Business, v.62, n.3, p.393-416, 1989.

Performance measurement without benchmarks : an examination of mutual fund returns. Journal of Business, v.66, n.1, p.47-68, Jan. 1993.

HWANG, S-W.

Information quality and portfolio performance measures : the degree of robustness and empirical evidence. New York, 1988. Dissertation (Ph.D.) - New York University.

JENSEN, M. C.

The performance of mutual funds in the period 1945-1964. Journal of Finance, v.23, p.389-416, May 1968.

Optimal utilisation of market forecasts and the evaluation of investment performance. In: SZEGO; SHELL (Ed.). Mathematical models in investment and finance. North Holland : North Holland Press, 1972. p.310-335. 
KON, S. J.;

JEN, F. C.

Estimation of the time-varying systematic risk and performance for mutual fund portfolios : an application of switching regression. Journal of Finance, v.33, n.2, p.457-475, May 1978.

The investment performance of mutual funds : an empirical investigation of timing, selectivity and market efficiency. Journal of Business, v. 62, n. 2, p. 263-289, Apr. 1979.

LEE, C. F.;

JEN, F. C.

Effects of measurement errors on systematic risk and performance measure of a portfolio. Journal of Financial and Quantitative, p.299-312, June 1978.

LEE, C. F.;

RAHMAN, S.

Market timing, selectivity, and mutual fund performance : an empirical investigation. Journal of Business, v.63, n.2, p.261-278, 1990.

\section{LEVY, $\mathrm{H}$.}

The CAPM and the investment horizon. Journal of Portfolio Management, p.32-40, Winter 1981.

Measuring risk and performance over alternative investment horizons. Financial Analysts Journal, p.61-68, 1984.

\section{LINTNER, J.}

The valuation of risky assets and the selection of risky investments in stock portfolios and capital budgets. Review of Economics and Statistics, v.57, n.1, p.13-37, Feb. 1965.

PFLEIDERER, P.;

BHATTACHA-RYA, S.

A note on performance evaluation.
Technical Report. Stanford : Stanford University, n.714, Oct. 1983.

ROLL, R.

Ambiguity when performance is measured by the securities market line. Journal of Finance, v.33, n.4, p.1051-1064, Sept. 1978.

Performance evaluation and benchmark errors (I). Journal of Portfolio Management, p. 05-12, 1980.

Performance evaluation and benchmark errors (II). Journal of Portfolio Management, p.17-22, 1981.

SANTOS, C. M.;

ARMADA, M. J. R.

Assimetria de informação e avaliação da performance de gestores de investimentos. In : XX ENCONTRO ANUAL DA ANPAD (1996 : Rio das Pedras). Anais ... ANPAD : Rio de Janeiro, 1996.

SHARPE, W. F.

Capital asset prices : a theory of market equilibrium under conditions of risk. Journal of Finance, v.19, n.3, p.425-442, 1964.

Mutual fund performance. Journal of Business, v.39, p.119138, Jan. 1966.

TREYNOR, J. L.

How to rate management of investment funds. Harvard Business Review, v.43, p.63-75, 1965.

TREYNOR, J. L.;

MAZUY, K. K.

Can mutual funds outguess the market? Harvard Business Review, v.44, p.131-136, 1966. 


\section{Anexo 1}

\section{Esquema Prático para o Cálculo da PCM}

1. Os Pesos: $\mathrm{W}_{\mathrm{i}, \mathrm{t}}=\left(\sigma_{\mathrm{i}}^{2}-\left(\mathrm{R}_{\mathrm{i}, \mathrm{t}}-\overline{\mathrm{R}}_{\mathrm{i}}\right) \overline{\mathrm{R}}_{\mathrm{i}}\right) /\left(\mathrm{T} \sigma_{\mathrm{i}}^{2}\right)$

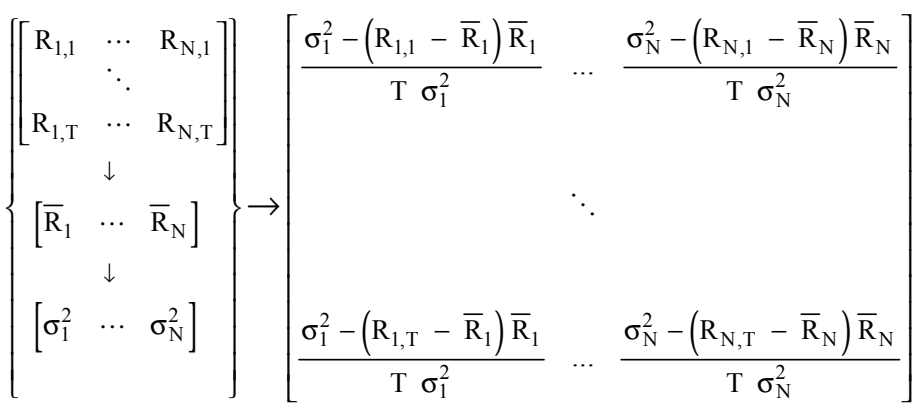

2. A Medida: $\mathrm{PCM}=\frac{1}{\mathrm{~T}-1} \sum_{\mathrm{t}=1}^{\mathrm{T}} \sum_{\mathrm{i}=1}^{\mathrm{N}}\left[\mathrm{R}_{\mathrm{i}, \mathrm{t}}\left(\mathrm{W}_{\mathrm{i}, \mathrm{t}}-\mathrm{W}_{\mathrm{i}, \mathrm{t}-1}\right)\right]$

$$
\begin{aligned}
& {\left[\begin{array}{ccc}
\mathrm{R}_{1,2} & \ldots & \mathrm{R}_{\mathrm{N}, 2} \\
& \ddots & \\
\mathrm{R}_{1, \mathrm{~T}} & \ldots & \mathrm{R}_{\mathrm{N}, \mathrm{T}}
\end{array}\right] \rightarrow\left[\begin{array}{ccc}
\mathrm{w}_{1,1} & \ldots & \mathrm{w}_{\mathrm{N}, 1} \\
& \ddots & \\
\mathrm{w}_{1, \mathrm{~T}} & \ldots & \mathrm{w}_{\mathrm{N}, \mathrm{T}}
\end{array}\right] \rightarrow\left[\begin{array}{lll}
\mathrm{R}_{1,2}\left(\mathrm{w}_{1,2}-\mathrm{w}_{1,1}\right) & \ldots & \mathrm{R}_{\mathrm{N}, 2}\left(\mathrm{w}_{\mathrm{N}, 2}-\mathrm{w}_{\mathrm{N}, 1}\right) \\
& \ddots & \\
& & \\
\mathrm{R}_{1, \mathrm{~T}}\left(\mathrm{w}_{1, \mathrm{~T}}-\mathrm{w}_{1, \mathrm{~T}-1}\right) & \ldots & \mathrm{R}_{\mathrm{N}, \mathrm{T}}\left(\mathrm{w}_{\mathrm{N}, \mathrm{T}}-\mathrm{w}_{\mathrm{N}, \mathrm{T}-1}\right)
\end{array}\right] \rightarrow\left[\begin{array}{c}
\sum_{\mathrm{i}} \\
\ldots \\
\sum_{\mathrm{i}}
\end{array}\right]} \\
& {\left[\sum_{\mathrm{t}}\right] /(\mathrm{T}-1)}
\end{aligned}
$$




\section{Anexo 2}

\section{Resumo das Características da Base de Dados}

\begin{tabular}{|c|c|c|c|c|c|c|c|c|c|c|c|c|}
\hline & & $1990-1992$ & & & 1993-1993 & & & 1990 & & & 1991 & \\
\hline Fundo & Períodos & $\mathrm{N}^{0}$ Acções & $\mathrm{N}^{\circ}$ Obs. & Períodos & $\mathrm{N}^{\circ}$ Acções & $\mathrm{N}^{\circ}$ Obs. & Períodos & $\mathrm{N}^{\mathrm{o}}$ Acções & $\mathrm{N}^{\circ}$ Obs. & Períodos & $\mathrm{N}^{\mathrm{o}}$ Acções & $\mathrm{N}^{\circ}$ Obs. \\
\hline FIP & 72 & 82 & 5904 & 48 & 47 & 2256 & 24 & 54 & 1296 & 24 & 39 & 936 \\
\hline INV & 72 & 93 & 6696 & 48 & 53 & 2544 & 24 & 73 & 1752 & 24 & 59 & 1416 \\
\hline MUL & 72 & 61 & 4392 & 48 & 41 & 1968 & 24 & 47 & 1128 & 24 & 41 & 984 \\
\hline PRI & 72 & 67 & 4824 & 48 & 54 & 2592 & 24 & 47 & 1128 & 24 & 43 & 1032 \\
\hline UNI & 72 & 67 & 4824 & 48 & 60 & 2880 & 24 & 39 & 936 & 24 & 35 & 840 \\
\hline VAL & 72 & 33 & 2376 & 48 & 18 & 864 & 24 & 28 & 672 & 24 & 9 & 216 \\
\hline
\end{tabular}

\begin{tabular}{|c|c|c|c|c|c|c|c|c|c|}
\hline \multirow[b]{2}{*}{ Fundo } & \multicolumn{3}{|c|}{1992} & \multicolumn{3}{|c|}{1993} & \multicolumn{3}{|c|}{1994} \\
\hline & Períodos & $\mathrm{N}^{\circ}$ Acções & $\mathrm{N}^{\circ}$ Obs. & Períodos & $\mathrm{N}^{0}$ Acções & $\mathrm{N}^{\circ}$ Obs. & Períodos & $\mathrm{N}^{0}$ Acções & $\mathrm{N}^{\circ}$ Obs. \\
\hline FIP & 24 & 43 & 1032 & 24 & 39 & 936 & 24 & 31 & 744 \\
\hline INV & 24 & 55 & 1320 & 24 & 49 & 1176 & 24 & 29 & 696 \\
\hline MUL & 24 & 33 & 792 & 24 & 33 & 792 & 24 & 32 & 768 \\
\hline PRI & 24 & 42 & 1008 & 24 & 40 & 960 & 24 & 42 & 1008 \\
\hline UNI & 24 & 33 & 792 & 24 & 44 & 1056 & 24 & 49 & 1176 \\
\hline VAL & 24 & 6 & 144 & 24 & 8 & 192 & 24 & 17 & 408 \\
\hline
\end{tabular}




\section{Anexo 3 \\ Montante Investido em Acções por Fundo}

\begin{tabular}{|c|c|c|c|c|c|c|c|c|}
\hline \multirow[b]{2}{*}{ Fundo } & \multicolumn{2}{|c|}{ 1990-1994 } & \multicolumn{2}{|c|}{ 1990-1992 } & \multicolumn{2}{|c|}{ 1993-1994 } & \multicolumn{2}{|c|}{1990} \\
\hline & Média & D.Padrão & Média & D.Padrão & Média & D.Padrão & Média & D.Padrão \\
\hline FIP & 1220826 & 301932 & 1282973 & 376632 & 1145105 & 133532 & 1741467 & 243976 \\
\hline INV & 1448607 & 1095997 & 2121988 & 992696 & 491972 & 194613 & 3355972 & 674406 \\
\hline MUL & 771556 & 176792 & 846290 & 117866 & 665746 & 198582 & 899631 & 132121 \\
\hline PRI & 528960 & 169448 & 536297 & 194227 & 525734 & 134064 & 705827 & 244428 \\
\hline UNI & 595206 & 291723 & 717616 & 323054 & 426406 & 121283 & 1105456 & 192724 \\
\hline VAL & 200471 & 180612 & 177736 & 180233 & 244887 & 187707 & 373485 & 188059 \\
\hline
\end{tabular}

Valores em Milhares de Escudos

\begin{tabular}{|c|c|c|c|c|c|c|c|c|}
\hline \multirow[b]{2}{*}{ Fundo } & \multicolumn{2}{|c|}{1991} & \multicolumn{2}{|c|}{1992} & \multicolumn{2}{|c|}{1993} & \multicolumn{2}{|c|}{1994} \\
\hline & Média & D.Padrão & Média & D.Padrão & Média & D.Padrão & Média & D.Padrão \\
\hline FIP & 1185676 & 54951 & 921776 & 99881 & 1045990 & 99736 & 1244219 & 77381 \\
\hline INV & 1755405 & 230440 & 1254586 & 140903 & 635872 & 155443 & 348072 & 100086 \\
\hline MUL & 773910 & 64174 & 865329 & 111790 & 794084 & 69346 & 537409 & 203475 \\
\hline PRI & 479044 & 77558 & 424022 & 64248 & 407889 & 81279 & 643578 & 33752 \\
\hline UNI & 653446 & 56233 & 393946 & 106250 & 319670 & 62358 & 533143 & 48928 \\
\hline VAL & 124903 & 23052 & 34819 & 13004 & 87671 & 83121 & 402103 & 116220 \\
\hline
\end{tabular}




\section{Anexo 4}

Bilhetes do Tesouro - Valores Médios Quinzenais

\begin{tabular}{|lccc|ccccc|}
\hline & $\mathbf{1 9 9 0 - 1 9 9 4}$ & $\mathbf{1 9 9 0 - 1 9 9 2}$ & $\mathbf{1 9 9 3 - 1 9 9 4}$ & $\mathbf{1 9 9 0}$ & $\mathbf{1 9 9 1}$ & $\mathbf{1 9 9 2}$ & $\mathbf{1 9 9 3}$ & $\mathbf{1 9 9 4}$ \\
\hline Média & $0.61 \%$ & $0.71 \%$ & $0.47 \%$ & $0.70 \%$ & $0.74 \%$ & $0.67 \%$ & $0.51 \%$ & $0.44 \%$ \\
D.Padrão & $0.12 \%$ & $0.04 \%$ & $0.06 \%$ & $0.02 \%$ & $0.01 \%$ & $0.05 \%$ & $0.06 \%$ & $0.04 \%$ \\
Máx. & $0.75 \%$ & $0.75 \%$ & $0.62 \%$ & $0.72 \%$ & $0.75 \%$ & $0.74 \%$ & $0.62 \%$ & $0.53 \%$ \\
Min. & $0.40 \%$ & $0.58 \%$ & $0.40 \%$ & $0.65 \%$ & $0.71 \%$ & $0.58 \%$ & $0.44 \%$ & $0.40 \%$ \\
\hline
\end{tabular}

\section{Anexo 5}

Índice BVL - Valores Médios Quinzenais

\begin{tabular}{|lccc|ccccc|}
\hline \multicolumn{2}{|c}{$\mathbf{1 9 9 0 - 1 9 9 4}$} & $\mathbf{1 9 9 0 - 1 9 9 2}$ & $\mathbf{1 9 9 3 - 1 9 9 4}$ & $\mathbf{1 9 9 0}$ & $\mathbf{1 9 9 1}$ & $\mathbf{1 9 9 2}$ & $\mathbf{1 9 9 3}$ & $\mathbf{1 9 9 4}$ \\
\hline Média & $0.03 \%$ & $-0.70 \%$ & $1.12 \%$ & $-1.61 \%$ & $-0.06 \%$ & $-0.48 \%$ & $1.84 \%$ & $0.40 \%$ \\
D.Padrão & $2.87 \%$ & $2.57 \%$ & $2.97 \%$ & $2.62 \%$ & $2.93 \%$ & $1.91 \%$ & $3.20 \%$ & $2.59 \%$ \\
Máx. & $9.18 \%$ & $9.18 \%$ & $8.72 \%$ & $5.32 \%$ & $9.18 \%$ & $2.19 \%$ & $8.72 \%$ & $5.68 \%$ \\
Min. & $-7.42 \%$ & $-7.42 \%$ & $-6.37 \%$ & $-7.42 \%$ & $-4.31 \%$ & $-6.21 \%$ & $-4.02 \%$ & $-6.37 \%$ \\
\hline
\end{tabular}

\section{Anexo 6}

\section{Ordenação dos Fundos para os Períodos em Análise}

\begin{tabular}{|c|c|c|c|c|c|c|c|c|}
\hline Fundos & 1990-1994 & 1990-1992 & 1993-1994 & 1990 & 1991 & 1992 & 1993 & 1994 \\
\hline FIP & 4 & 4 & 5 & 4 & 4 & 3 & 5 & 4 \\
\hline INV & 1 & 1 & 2 & 1 & 3 & 2 & 1 & 6 \\
\hline MUL & 3 & 5 & 4 & 5 & 5 & 1 & 4 & 2 \\
\hline PRI & 2 & 3 & 3 & 2 & 2 & 4 & 3 & 1 \\
\hline UNI & 5 & 2 & 6 & 3 & 1 & 5 & 6 & 5 \\
\hline VAL & 6 & 6 & 1 & 6 & 6 & 6 & 2 & 3 \\
\hline
\end{tabular}




\section{Anexo 7}

\section{Coeficiente de Correlação $\left(R_{s}\right)$ entre a Ordenação dos Fundos}

\begin{tabular}{|c|c|c|c|c|c|c|c|c|}
\hline & 1990-1994 & 1990-1992 & 1993-1994 & 1990 & 1991 & 1992 & 1993 & 1994 \\
\hline 1990-1994 & 1 & & & & & & & \\
\hline 1990-1992 & 0.60 & 1 & & & & & & \\
\hline 1993-1994 & 0.14 & -0.26 & 1 & & & & & \\
\hline 1990 & 0.77 & 0.94 & -0.09 & 1 & & & & \\
\hline 1991 & 0.31 & 0.83 & -0.54 & 0.77 & 1 & & & \\
\hline 1992 & 0.71 & 0.20 & -0.14 & 0.26 & -0.09 & 1 & & \\
\hline 1993 & 0.43 & 0.03 & 0.94 & 0.20 & -0.37 & 0.09 & 1 & \\
\hline 1994 & -0.03 & -0.60 & 0.14 & -0.37 & -0.26 & -0.03 & -0.03 & 1 \\
\hline
\end{tabular}

